\title{
Folding and Deployment of Closed Cross-Section Dual-Matrix Composite Booms
}

\author{
M. Sakovsky* and S. Pellegrino* \\ California Institute of Technology, Pasadena, CA 91125 \\ H.M.Y.C. Mallikarachchi ${ }^{\ddagger}$ \\ University of Moratuwa, Katubedda, Sri Lanka
}

\begin{abstract}
A dual-matrix composite boom is proposed as a way of realizing a deployable closed cross-section boom that is stiff, lightweight, and can be packaged in small volumes. Little work exists studying the folding and deployment behavior of closed cross-section boom made of composite shells and this paper addresses this by investigating the behavior for two closed cross-section designs. Experimental techniques for measuring the folded shape of curved shells undergoing large deformations is presented. Furthermore, experimental measurements of the moment-rotation response of the two booms are discussed. A study using commercially available finite element software yields simulation techniques for successfully predicting the folded shape of closed-cross section booms. The drawbacks of the software when predicting the moment-rotation response are addressed. The application of these techniques for the chosen designs demonstrate that dual-matrix booms are a promising alternative to existing composite deployable booms.
\end{abstract}

\section{Introduction}

Large apertures in space, including reflector antennas, solar arrays, and telescope primary mirrors, require the use of deployable structures to comply with the volume limitations imposed by modern launch vehicle fairings. One method of realizing such structures is through the use of rigid elements connected by mechanical hinges, as is done with the Astromesh reflector from Northrop Grumman. ${ }^{1}$ However, mechanical hinges require actuation using motors or springs and hence tend to be heavy and complex. An alternate approach is the use of shell structures that deform elastically in order to be packaged. The resulting structure is lightweight, can be deployed using stored strain energy, and eliminates the use of complex mechanical hinges. However, folding of these shell structures is constrained to their elastic limits. Deployable shell structures include both open and closed cross-section booms which can be flattened and packaged around a central hub. The simplest example is the storable tubular extendible member (STEM) boom which has been widely used in space structure since $1988 .^{2}$ An example of a closed cross-section boom is the DLR CFRP boom. ${ }^{3}$

Dual-matrix composites have been previously proposed for use in large aperture space structures as a way of tackling the limitations of mechanical hinges and foldable shell structures. Dual-matrix composites contain a continuous fiber reinforcement with a soft, elastomer matrix in specific embedded hinge regions and a traditional stiff matrix elsewhere, as shown in Figure 1. The elastomer matrix can fold elastically to high strains, allowing folds of as much as $180^{\circ}$. Furthermore, fiber damage during folding is prevented as the fibers on the compression side of the fold undergo microbuckling., ${ }^{4,5}$

\footnotetext{
${ }^{*}$ Graduate Student, Graduate Aerospace Laboratories, 1200 East California Boulevard, Mail Code 105-50. e-mail: msakovsk@caltech.edu

${ }^{\dagger}$ Professor of Aeronautics and Civil Engineering, Graduate Aerospace Laboratories, 1200 East California Boulevard, Mail Code 301-46, Fellow AIAA. e-mail: sergiop@caltech.edu

${ }^{\ddagger}$ Senior Lecturer, Department of Civil Engineering, University of Moratuwa, Sri Lanka, and Member AIAA. e-mail: yasithcm@uom.1k
} 


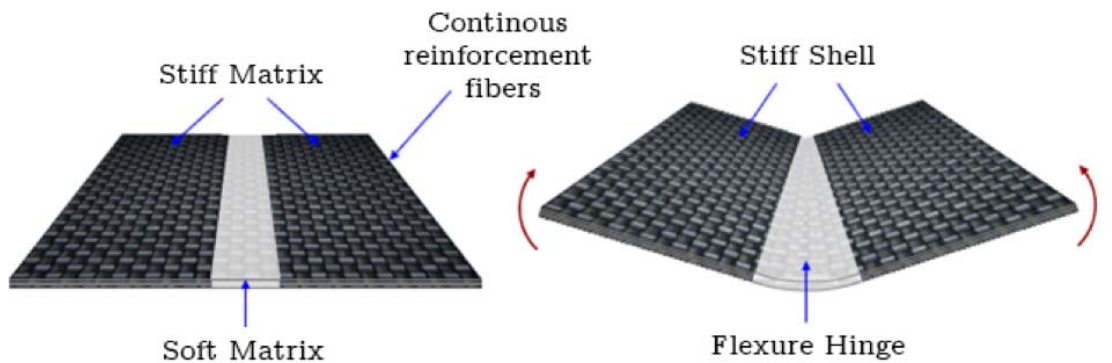

Figure 1. A schematic of a dual-matrix composite hinge

Dual-matrix composites have been first addressed by L'Garde and their functionality demonstrated through the fabrication of a flat sheet packaged using the Miura-Ori origami pattern. ${ }^{6}$ More recently, it has been shown that these composites can be folded to very complex shapes, such as an origami crane. ${ }^{7}$ Furthermore, a functioning high-performance conical log spiral antenna for CubeSats has been constructed using dual-matrix composites, demonstrating that these materials can be used to fabricate lightweight, strain energy deployable structures. ${ }^{8}$

Despite their numerous applications, there has been no detailed study of the folding and deployment behavior of dual-matrix composites. More significantly, little work has been done towards the study of folding and deployment of closed cross-section booms composed of shell structures. This work tackles this gap through the application of dual-matrix composites to deployable booms where the hinges allow the boom to flatten and fold. A sample application to CubeSat deployable payloads is considered (see Figure 2). For simplicity, the folding and deployment problem considered here is that of a single $90^{\circ}$ fold at the center of the boom. However, this folding scheme is not unique as the entire boom can also be flattened and packaged around a hub as is done with STEM and the DLR booms.

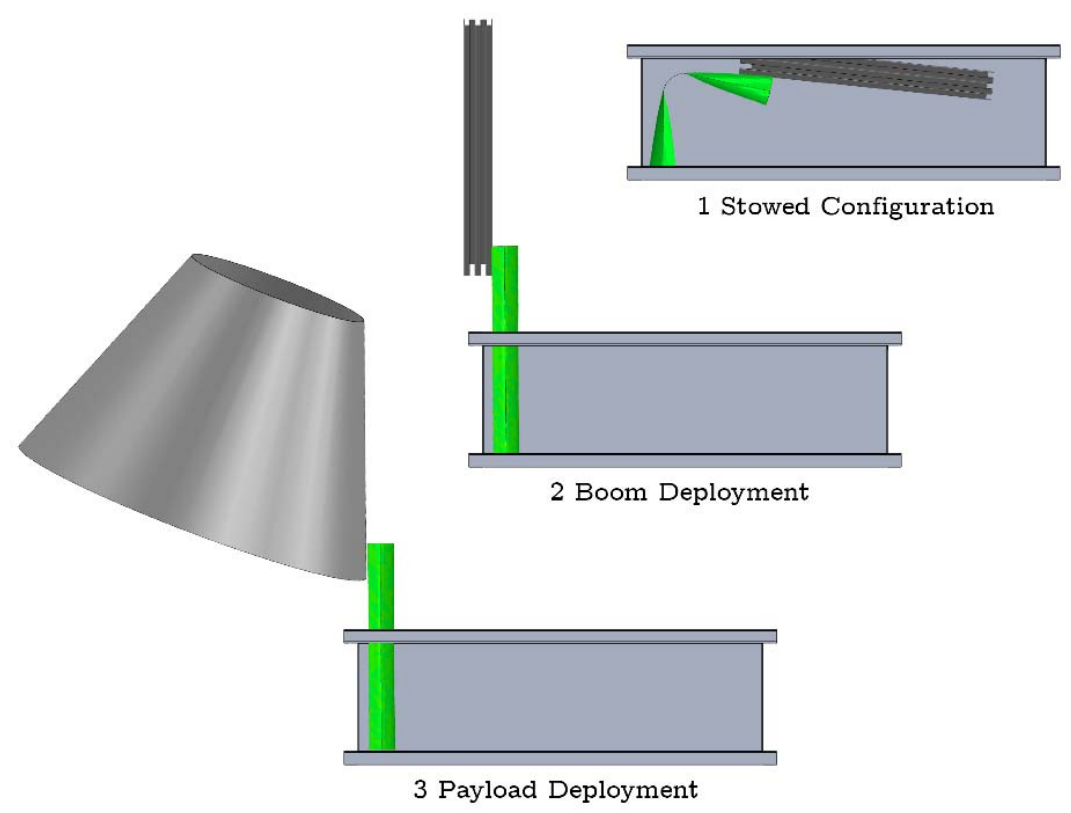

Figure 2. Application of dual-matrix boom to CubeSat deployable structures (boom is shown in green)

This work uses two closed cross-section booms designs to develop experimental and numerical techniques for the study of folding and deployment of closed cross-section booms. Section II presents the boom de- 
signs studied in this paper and describes the fabrication of prototypes. Section III discusses experimental measurement of the moment-rotation response for the two booms. Section IV presents techniques used to measure the folded shape of the booms. Section V describes the development of finite element simulation techniques for predicting the folded shape and moment-rotation response. These techniques are applied to the two designs and results are compared to experiments. Section VI concludes the paper and identifies future work.

\section{Boom Geometry and Fabrication}

This study investigates two types of closed cross-section deployable booms. First, a dual-matrix boom with two $8 \mathrm{~mm}$ wide diametrically opposite silicone hinges (see Figure 3(a)). Second, a single-matrix boom composed of two composite tape-springs connected by tape along the edges, to simulate a moment-less connection (see Figure 3(b)). The second configuration is used to isolate the effects of the silicone hinges on the deployment behavior of the boom.

(a)
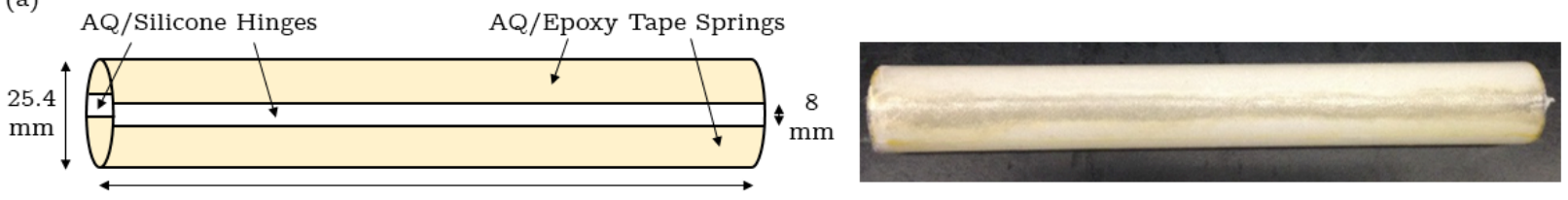

(b)
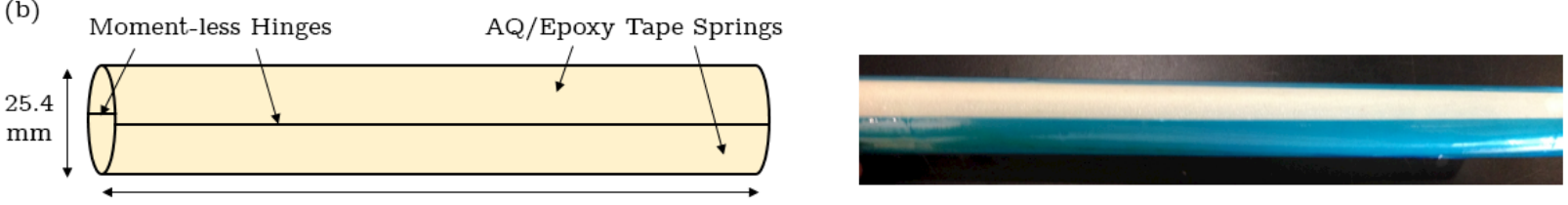

Figure 3. Two closed cross-section boom geometries studied (a) dual-matrix boom (b) single-matrix boom

Sample booms have been fabricated using Astroquartz (AQ) II plain-weave (p-w) fabric, Loctite 5055 UV-cure silicone for the foldable hinges, and PMT-F4B epoxy from Patz Technologies for the stiff panels. A quasi-isotropic 3-ply symmetric $[45 / 0 / 45]_{\mathrm{p}-\mathrm{w}}$ layup was used. The booms were fabricated following the procedure presented in Ref. ${ }^{8,9}$ It was found that manufacturing defects, such as dry fabric and wrinkles, resulted in the formation of sharp kinks and broken fibers as the booms were folded. Therefore, modifications were required to existing procedures to reduce defects. Most notably, heat shrink was applied to the uncured booms before applying the vacuum bag to prevent wrinkle formation during the autoclave cure process. The updated fabrication process is shown in Figure 4. The single-matrix boom was fabricated with the same layup and used a similar procedure.

\section{Moment-Rotation Response}

An important characteristic of boom folding and deployment is the moment-rotation response as it gives an indication of how the boom will deploy and whether there is a possibility of the boom not latching. For both boom configurations shown in Figure 3, the moment-rotation response is expected to resemble that of two tape-springs bending together, one in equal-sense and one in opposite-sense bending. The folding angle, $\theta$, is defined as shown in Figure 5(a). Increasing the fold angle, a steep linear region for small angles is observed followed by a sharp drop in moment. This is followed by a region of constant moment. Moreover, a slight increase in moment is expected at high folding angles due to the interaction of the two tape springs. This response is illustrated in Figure 5(c).

To experimentally characterize the moment-rotation response, the ends of each boom were rigidly attached to the two arms of the apparatus shown in Figure 6. The arm on the right was fixed, while the one on the was left free to slide smoothly along a linear guide bearing. The rotation of each arm was adjusted using gears. Strain gauges were used to measure the strain imparted by the boom on each arm.

The boom was pinched at the centre to initiate folding and was brought to a fully folded configuration 


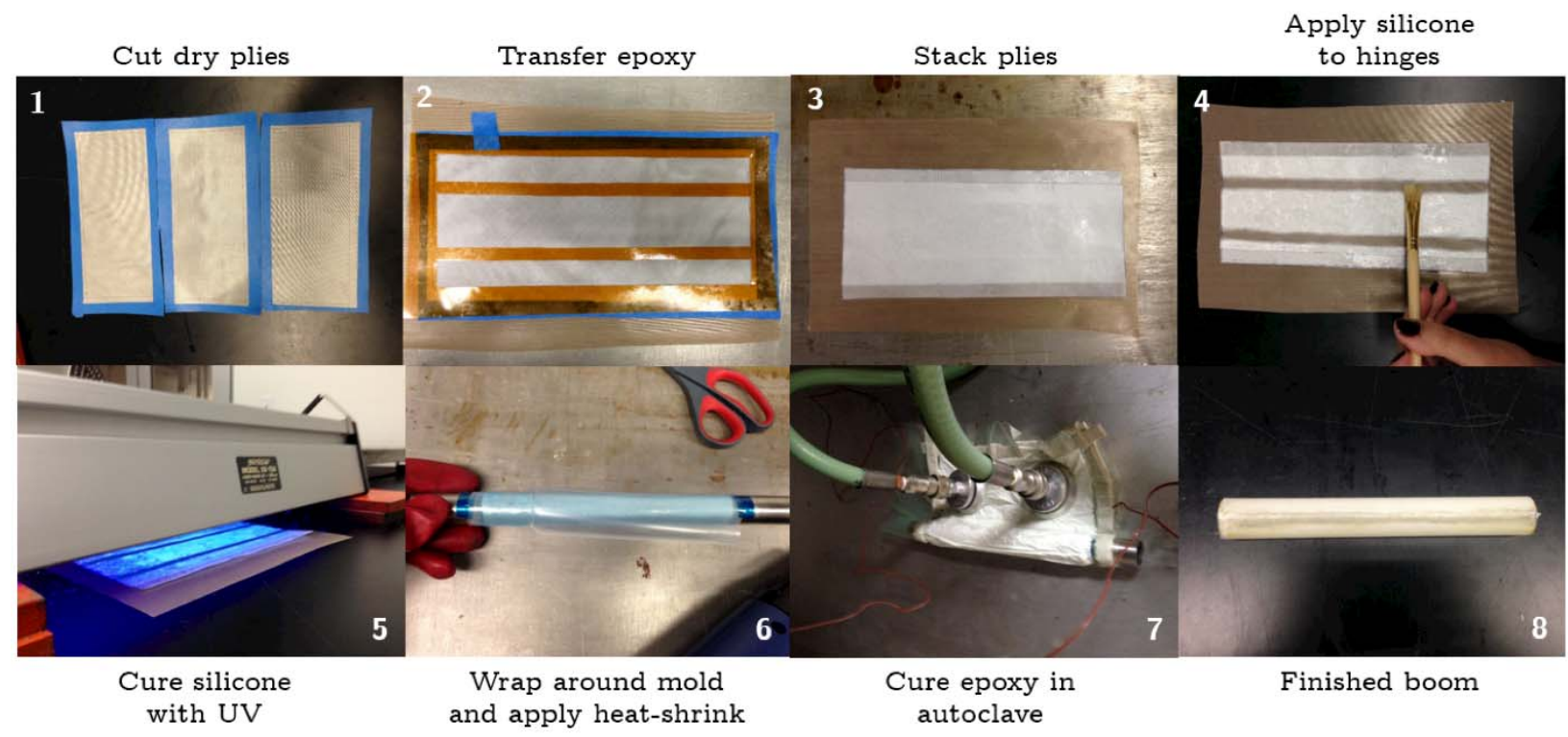

Figure 4. Steps in the manufacture of dual-matrix composite booms

(a)

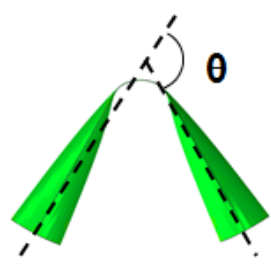

(b)

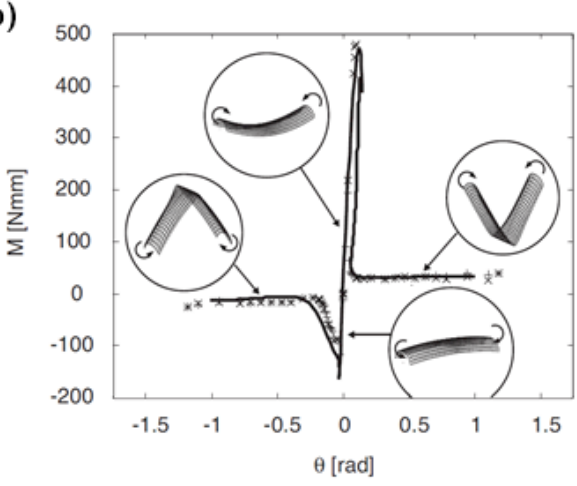

(c)

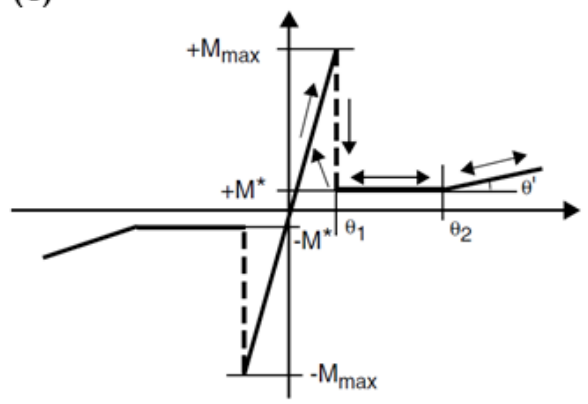

Figure 5. Moment-angle relationship for tape springs (a) definition of fold angle, $\theta$ (b) moment-angle relationship for a single tape spring ${ }^{13}$ (c) moment-angle relationship for folding the two tape spring configuration in $(\mathrm{a})^{13}$

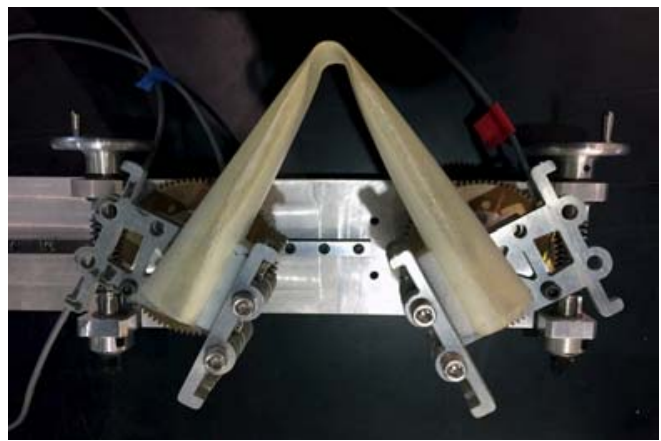

Figure 6. Apparatus for measuring the moment-rotation response of booms 
corresponding to $\theta \approx 120^{\circ}$. The boom was deployed quasi-statically and the fold angle and strain measured approximately every $5^{\circ}$ for $\theta>40^{\circ}$ and every $1^{\circ}$ for $\theta<40^{\circ}$ in order to accurately capture the peak moment.

Figure 7 shows the moment-rotation response obtained for the single-matrix and dual-matrix booms, respectively. The response of both samples exhibits the expected constant moment region with a sharp moment peak at low angles. Table 1 shows a comparison of characteristic values for the two booms, where the steady-state moment value represents the mean moment measured for $\theta \in\left[50^{\circ}, 100^{\circ}\right]$ averaged over three nominally identical tests. The silicone hinges contribute a significant amount to the strain energy stored in the boom, increasing the peak moment by $33 \%$ and the steady-state moment by $147 \%$. Furthermore, the peak angle is slightly reduced for the dual-matrix booms.

(a)

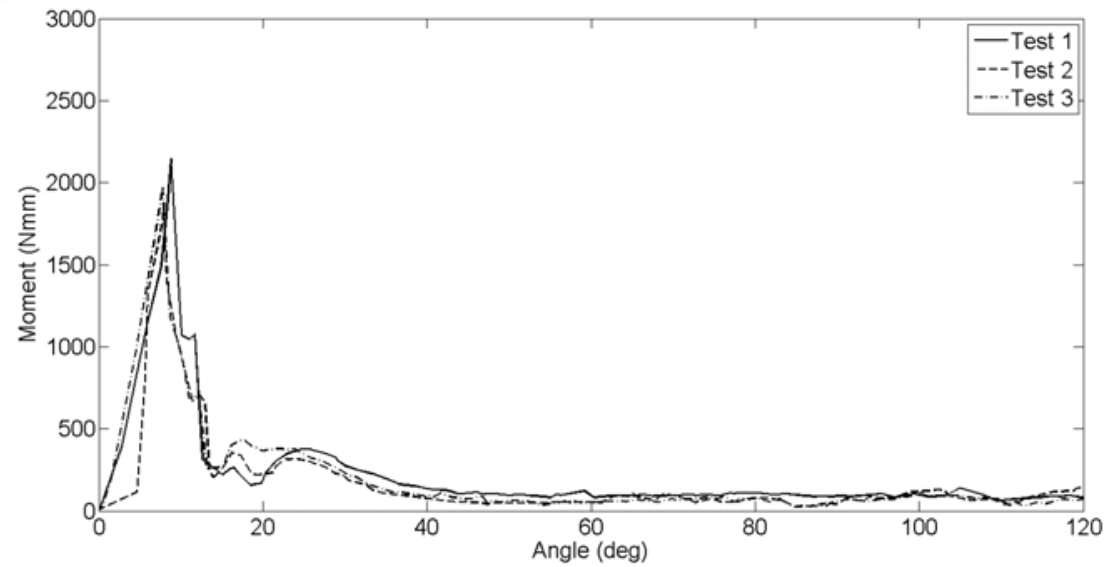

(b)

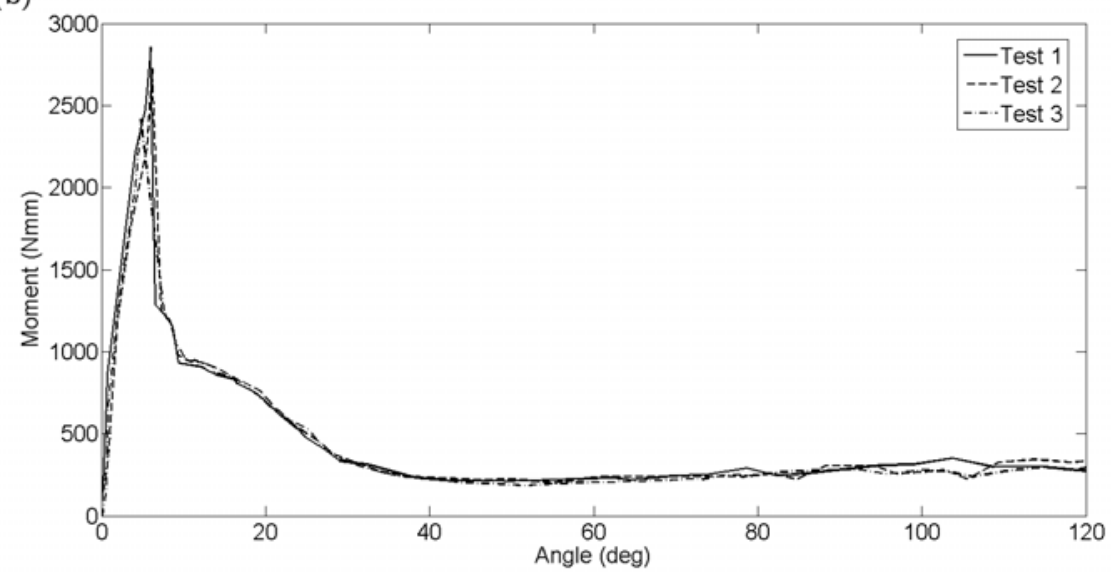

Figure 7. Experimental moment-angle relationship (a) single-matrix boom (b) dual-matrix boom

\begin{tabular}{cccc} 
& $\begin{array}{c}\text { Steady-state Moment } \\
(\mathbf{N m m})\end{array}$ & $\begin{array}{c}\text { Peak Moment } \\
(\mathbf{N m m})\end{array}$ & $\begin{array}{c}\text { Peak Angle } \\
(\mathbf{d e g})\end{array}$ \\
\hline Single-Matrix Boom & 100 & 2150 & 8.8 \\
Dual-Matrix Boom & 247 & 2850 & 6.0 \\
\hline
\end{tabular}

Table 1. Comparison of moment-rotation responses for single-matrix and dual-matrix booms

The effects of the silicone are most prominent at low angles as the silicone hinges smooth out the deployment process. The single-matrix boom response shows two distinct peaks corresponding to the deployment of the two tape springs. The distinct peaks form as the outer tape spring, undergoing equal-sense bending, deploys first. The dual-matrix boom response shows only a single peak, as the smaller peak is smoothed out by the silicone hinges. 
Theoretical predictions for the steady-state moment can be obtained from the bending stiffness matrix of the 3-ply AQ/epoxy composite. ${ }^{13}$ For two tape springs bending together, one in the equal sense and one in the opposite sense, with no connection between them, the predicted steady-state value is $15 \mathrm{Nmm}$. This value is $85 \%$ and $94 \%$ lower than the response measured for the single-matrix and dual-matrix boom, respectively. This demonstrates the importance of the closed cross-section.

\section{Boom Shape Measurements}

Quasi-static measurements of the booms' fold regions were performed using digital image correlation (DIC). Two Grasshopper 50S5M-C cameras from Point-Grey Research each equipped with a Xenoplan $\mathrm{f} / 1.9-35 \mathrm{~mm}$ lens from Schneider-Kreuznach were used. The stereo pair was positioned $50 \mathrm{~cm}$ from the boom to image the fold region of the outer tape-spring. A narrow aperture of $\mathrm{f} / 16$ was used creating a wide depth of field to keep the fold in focus for all angles. For a $35 \mathrm{~mm}$ lens, it is recommended to keep a stereo angle of $20^{\circ}<\alpha<60^{\circ}$, with larger angles favorable to reducing error. ${ }^{10}$ A stereo angle of $\alpha=40^{\circ}$ was used as it was found that lower angles resulted in large correlation errors. A schematic of the setup is shown in Figure 8. The test boom was attached to the apparatus described in Section III in order to measure the folded shape at various folding and deployment angles.
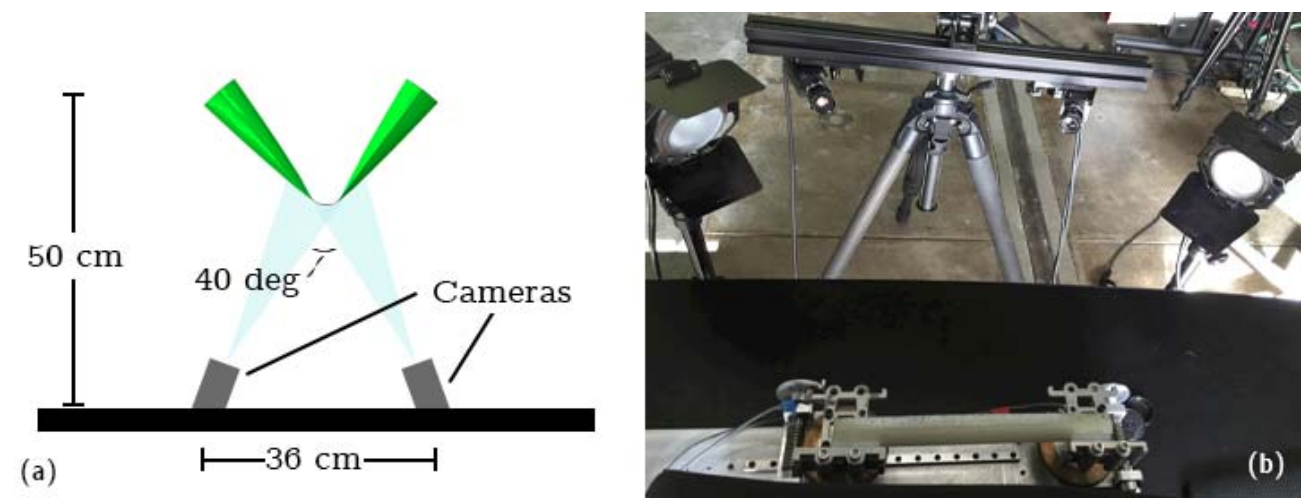

Figure 8. DIC setup for shape measurement experiments (a) schematic of the geometry used for the setup (b) photograph of the setup

The stereo cameras were calibrated using 30 images of a $12 \times 9$ grid of dots with $6 \mathrm{~mm}$ spacing. A black and white speckle pattern was applied to the boom using spray paint. Images of the pattern on a fully deployed boom were used as a reference. The boom was pinched to initiate folding and images were taken approximately every $5^{\circ}$ until the boom was folded to $100^{\circ}$. The process was repeated for deployment. The curvatures in the fold region were computed using the Vic3D software from Correlated Solutions.

Figure 9 shows the variation of longitudinal curvature measured along the fold region for various fold angles for the single-matrix and dual-matrix booms. The fold region has analogous characteristics in the two booms, showing a highly localized region of longitudinal curvature. The curvature was averaged across a $13 \times 5 \mathrm{~mm}$ region centered on the vertex of the fold to extract fold radius as a function of the fold angle, $\theta$, see Figure 10. The slight discrepancy in the fold radius curves for folding and deployment can be explained by the fact that the localization that leads to the formation of the fold was initiated manually. The fold radius approaches a steady-state value and is approximately constant for folds of $90^{\circ}$ and greater. The steady-state fold radius is $10.8 \mathrm{~mm}$ for the single-matrix boom and $10.0 \mathrm{~mm}$ for the dual-matrix boom, a decrease of $7.4 \%$.

\section{Folding and Deployment Simulations}

\section{A. Simulation Techniques}

A finite element model of both booms was created using the ABAQUS/Explicit finite element software (see Figure 11). The AQ/epoxy composite was modeled using S3 and S4 shell elements with a fine mesh around the fold region and a coarser mesh towards the ends of the boom. The model also contained two hinge 


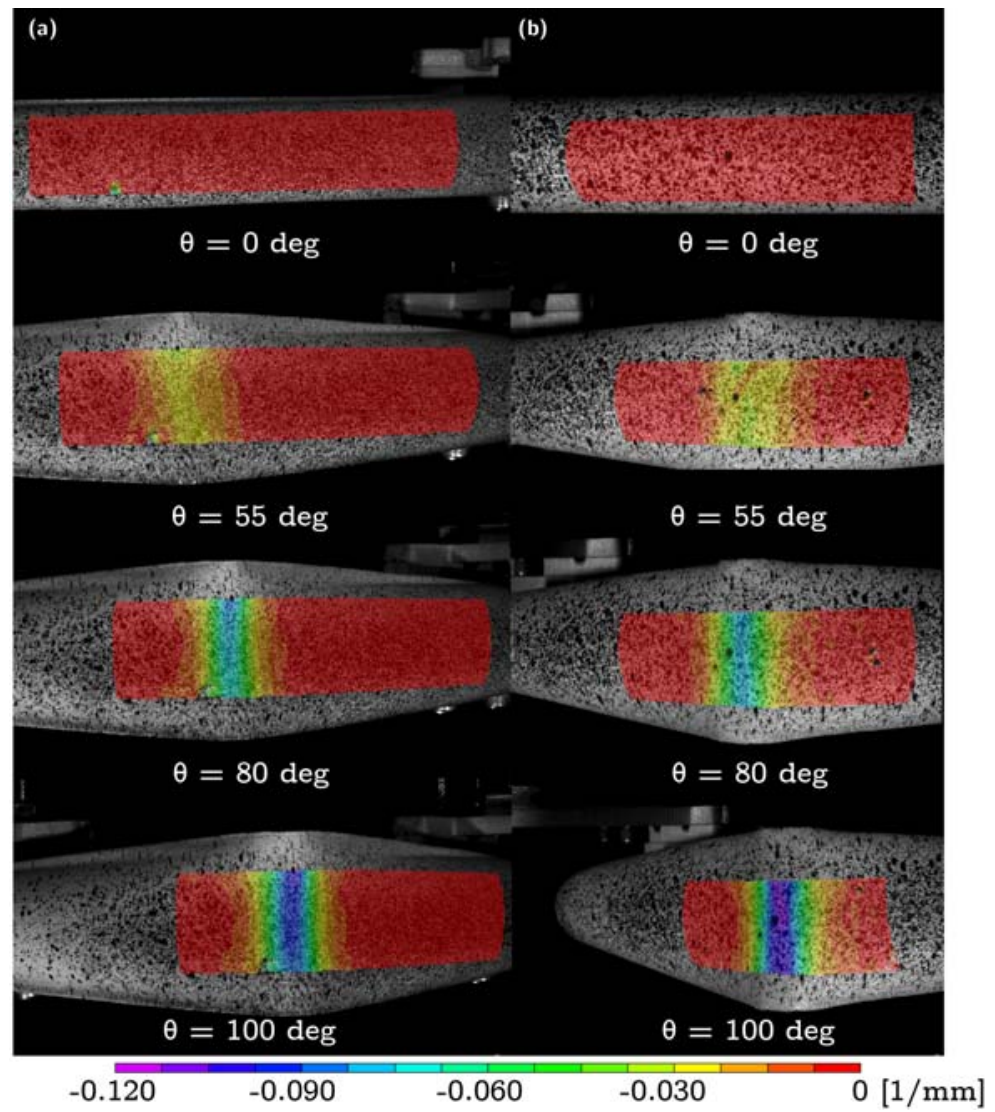

Figure 9. Variation of longitudinal curvature with fold angle (a) single-matrix boom (b) dual-matrix boom
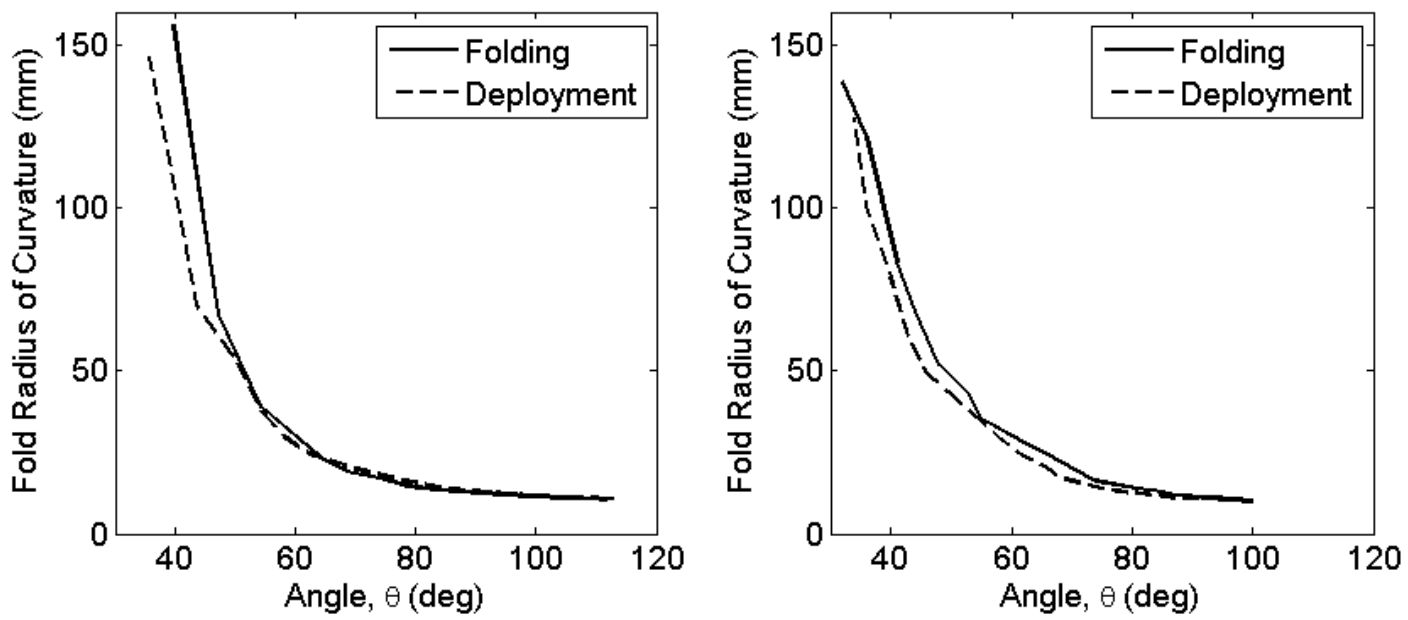

Figure 10. Variation of fold radius of boom during folding and deployment (a) single-matrix boom (b) dualmatrix boom 
regions. Modeling techniques for these are presented in subsequent sections. The material properties of the composites were defined in a cylindrical coordinate system (with directions $x, y$, and $z$ representing the longitudinal, circumferential, and radial coordinates, respectively) and applied using the ${ }^{*}$ Shell General Section keyword in ABAQUS. The ABD stiffness matrices used for the epoxy and silicone composites are given by,

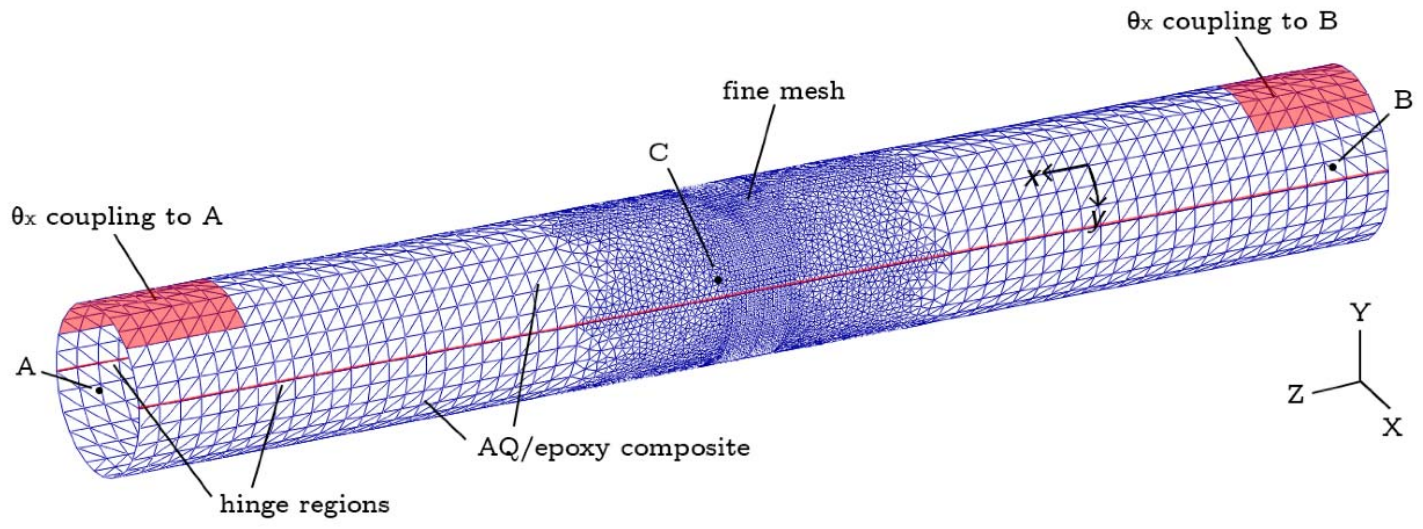

Figure 11. Finite element model of dual-matrix composite boom.

$$
A B D_{E}=\left(\begin{array}{ccccccc}
2569 & 972 & 0 & \mid & 0 & 0 & 0 \\
972 & 2569 & 0 & \mid & 0 & 0 & 0 \\
0 & 0 & 1128 & \mid & 0 & 0 & 0 \\
-- & -- & -- & -- & -- & -- & -- \\
0 & 0 & 0 & \mid & 4.3 & 2.4 & 0 \\
0 & 0 & 0 & \mid & 2.4 & 4.3 & 0 \\
0 & 0 & 0 & \mid & 0 & 0 & 2.7
\end{array}\right)
$$

and

$$
A B D_{S}=\left(\begin{array}{ccccccc}
1809 & 945 & 0 & \mid & 0 & 0 & 0 \\
945 & 1809 & 0 & \mid & 0 & 0 & 0 \\
0 & 0 & 945 & \mid & 0 & 0 & 0 \\
-- & -- & -- & -- & -- & -- & -- \\
0 & 0 & 0 & \mid & 6.2 & 5.8 & 0 \\
0 & 0 & 0 & \mid & 5.8 & 6.2 & 0 \\
0 & 0 & 0 & \mid & 0 & 0 & 5.8
\end{array}\right)
$$

where the units are $\mathrm{N}$ and $\mathrm{mm}$, for both matrices.

The in-plane stiffness was estimated using Classic Lamination Theory in combination with the Mosaic model. For thin three-ply composites, the Mosaic model is known to be inaccurate by as much as $100 \% .{ }^{12}$ Therefore, the bending stiffness used was obtained by measuring $D_{11}$ using a four point bending test on flat coupons made of the composite. The rest of the $D$ matrix was scaled by the ratio of the experimentally measured $D_{11}$ to the theoretical $D_{11}$.

The General Contact feature was assigned to the entire model by specifying Contact Inclusions, All Exterior. With this option ABAQUS/Explicit automatically defines potential contact surfaces around the whole hinge surface.

The boundary conditions imposed at various cross-sections of the boom can significantly affect the results of the simulations. ${ }^{11}$ To fold the boom, $10 \mathrm{~mm}$ long strips at the left and right ends were attached to reference nodes A and B, respectively, through ${ }^{*}$ Coupling with the ${ }^{*}$ Kinematic 4,4 option to couple the fourth degree of freedom, $\theta_{X}$ (see Figure 11). This allows the rotational boundary conditions to be specified at the reference nodes and ensures the boom cross-section is free to ovalize during folding. In order to simulate the equal end 
moment conditions imposed during experiments, the reference nodes $\mathrm{A}$ and $\mathrm{B}$ were attached to a dummy node, $\mathrm{C}$, using the ABAQUS command ${ }^{*}$ Equation, as prescribed by Equation 3, where $\theta_{X}$ denotes a rotation about the global $X$-axis.

$$
\theta_{X}^{A}-\theta_{X}^{B}=\theta_{X}^{C}
$$

To compute the folded configuration observed in Figure 6 the boom was pinched at the center using a pressure of $0.05 \mathrm{MPa}$ over $0.2 \mathrm{~s}$. To simulate the folding process the folding angle $\theta_{X}^{C}$ was incremented from $0^{\circ}$ to $115^{\circ}$ over a time interval of $1.5 \mathrm{~s}$. The folding sequence is visualized in Figure 12 . The deployment step reversed the folding process over a time interval of $4 \mathrm{~s}$.

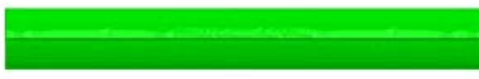

Undeformed

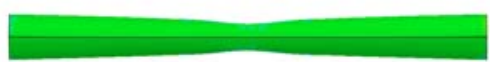

Pinched

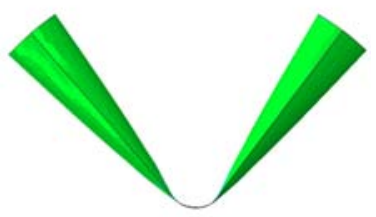

Folded

Figure 12. Folding sequence.

In order to obtain an accurate quasi-static moment-rotation response using a dynamic deployment step in ABAQUS several criteria were met. First, all loads were applied slowly and smoothly using the ${ }^{*}$ smooth step option. Next, the integration time step was kept as large as possible through the use of a time scaling factor, $\alpha$, subject to the Courant stability limit given by Equation 4 ,

$$
\Delta t=\alpha\left(\sqrt{1+\zeta^{2}}-\zeta\right) \frac{l_{\min }}{c_{d}}
$$

where $\zeta$ is the fraction of critical damping, $l_{\min }$ is the minimum element length, and $c_{d}$ is the wave speed.

Finally, numerical damping was used to reduce the kinetic energy to $\ll 10 \%$ of the internal energy of the boom. This was accomplished by applying a viscous, velocity dependent normal pressure, $p=-c_{v} \boldsymbol{v} \cdot \boldsymbol{n}$, to all elements on the outside of the boom. Here, $c_{v}$ is the magnitude of the numerical damping, $\boldsymbol{v}$ is the velocity, and $\boldsymbol{n}$ is the surface normal.

\section{B. Single-Matrix Boom}

\section{1. *Tie Constraint Hinge Model}

In modeling the single-matrix boom, each node along the hinge line was constrained to its counterpart on the opposing tape-spring in the three translational degrees of freedom using the ${ }^{*}$ Tie constraint in ABAQUS. This hinge represents the moment-less tape hinge holding the tape-springs together in the boom prototype. The model contains approximately 5,500 S3 shell elements.

As detailed in Section V.A, simulation of thin shell structures in a quasi-static manner requires a rigorous investigation of several simulation parameters. In this study, the effects of mesh size, viscous damping, time scaling factor, bulk viscosity damping, and rate of load application were investigated. The most critical parameters were found to be the mesh size and viscous damping.

A smaller mesh size ensures accuracy of a finite element solution but greatly increases simulation time and can cause stability issues. A mesh convergence study was done by refining the mesh until the solution converged. The ratio of the fine to coarse mesh spacing was kept constant between models and the fold radius was used as a measure of convergence. Table 2 shows the effects of mesh spacing on the fold radius at the end of the folding step. Refining the mesh beyond a minimum element size of $1 \mathrm{~mm}$ had little effect on the solution, with the slight variation of fold radius below the $1.0 \mathrm{~mm}$ mesh explained by the fact that viscous damping was not tuned for each mesh size. As a result a minimum mesh spacing of $1 \mathrm{~mm}$ is used to reduce simulation times.

A second critical parameter was found to be viscous damping, which allows kinetic energy to be kept low while maintaining short simulation times. However, large values of damping result in inaccuracies in the 


\begin{tabular}{r|ccccc} 
Smallest Element Size (mm) & 3.0 & 2.0 & 1.0 & 0.8 & 0.75 \\
\hline Fold Radius $(\mathbf{m m})$ & 33.33 & 9.09 & 15.03 & 14.42 & 14.59
\end{tabular}

Table 2. Effect of mesh refinement on boom fold radius

folded shape of the boom. The ABAQUS manual recommends that a damping coefficient of $c_{v} \approx 1-2 \% \rho c_{d}$. For this problem, this results in $c_{v}=0.2 \mathrm{~kg} \cdot \mathrm{mm}^{-1} \cdot \mathrm{s}^{-1}$. In practice, this value was found to be several orders of magnitude too large. Starting from the recommended value, the damping was decreased until the amount of kinetic energy in the system caused oscillations in the internal energy.
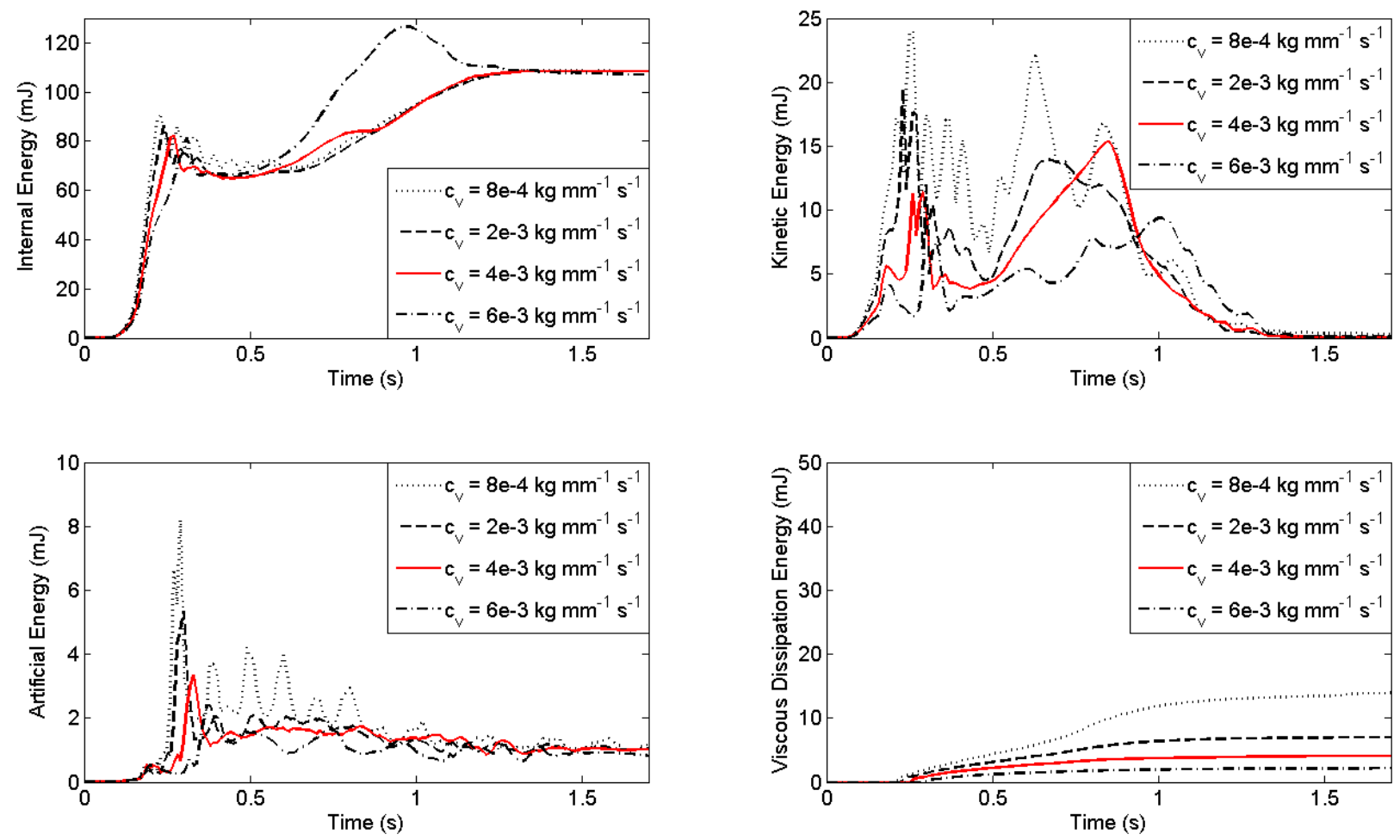

Figure 13. Evolution of internal, kinetic, artificial, and viscous dissipation energy as a function of viscous damping.

The evolution of the internal, kinetic, artificial, and viscous damping energy for various value of $c_{v}$ is shown in Figure 13. High values of damping cause the internal energy to overshoot its final value for the folded configuration while low value cause a buildup of viscous dissipation and large artificial and kinetic energies. A value of $c_{v}=4.0 \times 10^{-3} \mathrm{~kg} \cdot \mathrm{mm}^{-1} \cdot \mathrm{s}^{-1}$ was chosen as the optimal, indicated by the red curve in Figure 13. For this value, the kinetic energy is approximately zero in the fully folded configuration, indicating a stable configuration that can be used for deployment.

The folded shape for several values of damping is shown in Figure 14. It can be seen that a large value for damping results in a much greater than expected fold radius. A fold radius of $14.2 \mathrm{~mm}$ is achieved for the chosen value of damping.

Figure 15 shows the variation of the cross-section of the fully folded boom away from the center of the fold $(0 \mathrm{~mm})$. The two tape springs are tightly folded in the fold region (measured to be approximately 22 $\mathrm{mm}$ in length) and the cross-section opens up rapidly towards an ovalized shape, at the ends of the boom.

A repetition of the damping study for the deployment phase yield an optimal $c_{v}=1.0 \times 10^{-2} \mathrm{~kg} \cdot \mathrm{mm}^{-1} \cdot \mathrm{s}^{-1}$ to keep kinetic energy below $1 \%$ of the internal energy. Another crucial parameter in the deployment step was the bending stiffness of the AQ/Epoxy composite. When using the experimentally measured bending stiffness, the boom does not latch on deployment and remains in a pinched configuration. It was found that a bending stiffness of at least $D_{11}=10 \mathrm{Nmm}$ is required for the boom to latch, more than 2 times the 


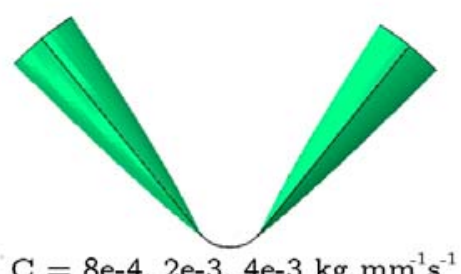

$\mathrm{C}_{\mathrm{v}}=8 \mathrm{e}-4,2 \mathrm{e}-3,4 \mathrm{e}-3 \mathrm{~kg} \mathrm{mm^{-1 }} \mathrm{s}^{-1}$

Fold Radius $=14.2 \mathrm{~mm}$

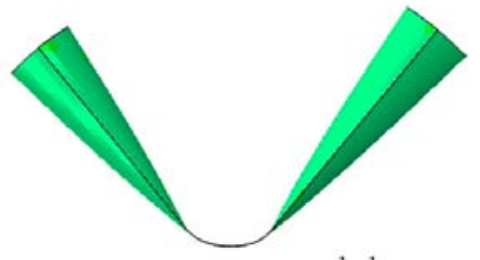

$\mathrm{C}_{\mathrm{v}}=6 \mathrm{e}-3 \mathrm{~kg} \mathrm{~mm} \mathrm{~s}^{-1}$

Fold Radius $=20.0 \mathrm{~mm}$

Figure 14. Fold radii obtained for different values of viscous damping.
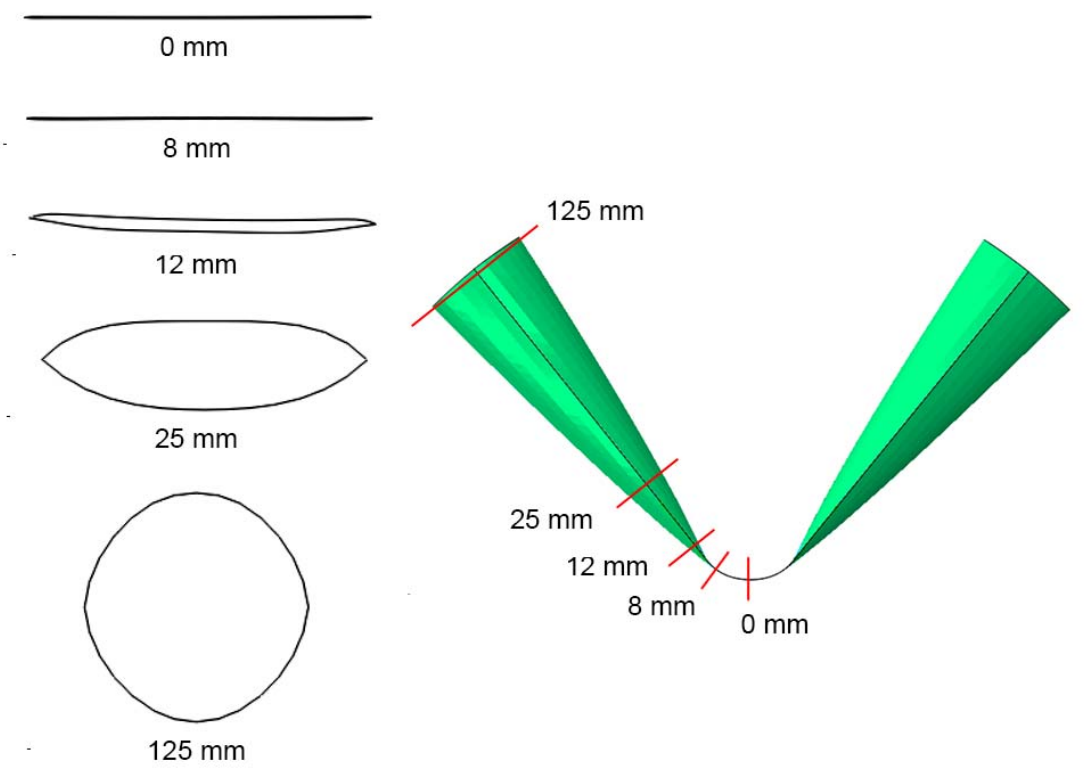

Figure 15. Cross-section of folded boom

11 of 18 
experimentally measured value.

To achieve deployment, a small outward pressure of $8 \mathrm{kPa}$ ( $15 \%$ of the pinching pressure used to fold the boom) was applied to both tape-springs around the fold region. This pressure was applied over a time interval of $0.5 \mathrm{~s}$ beginning at $40^{\circ}$ when the outer tape spring is about to latch. The kinetic energy is kept below $10 \%$ of the internal energy except at the instant the boom latches. However, artificial energy buildup during the latching to $5.6 \mathrm{~mJ}$ results in the same amount of residual strain energy at the end of deployment. Figure 16 shows a comparison of the simulated and experimentally obtained shapes for various angles. Overall, the shape during deployment agrees well with that seen experimentally, except that the simulated boom deploys at a higher angle than that observed experimentally.
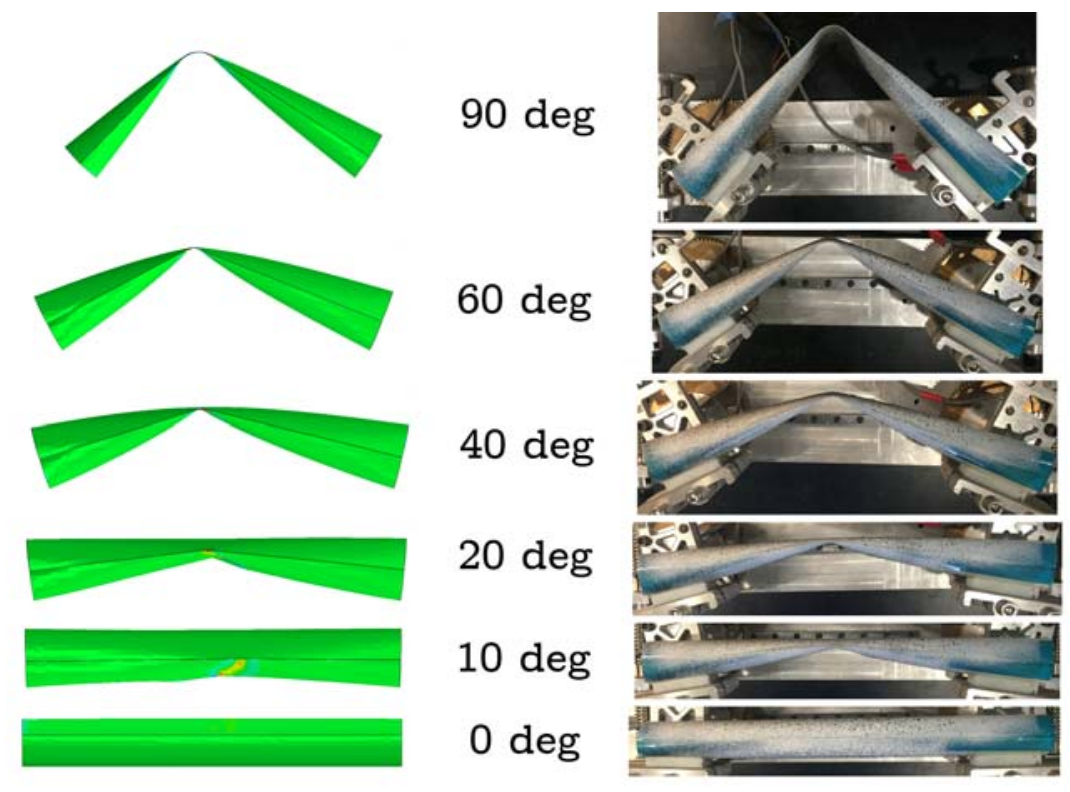

Figure 16. Comparison of experimentally observed and simulated deployments

The moment was extracted at the dummy point, C, and plotted against the deployment angle (see Figure 17). Note that the negative moment is a result of the pressure applied to deploy the boom. Qualitatively the curve matches that observed experimentally, exhibiting a steady state region and a sharp peak at $9^{\circ}$, which is close to the deployment angle observed experimentally. However, both the steady state moment and peak moment are significantly lower than measured (see Table 3). The simulated steady-state value is close to the theoretically predicted value of $15 \mathrm{Nmm}$ (Section III). Therefore, it can be concluded that this model is not able to capture the effect of the moment-less connection between the two tape-springs.

\begin{tabular}{rccc} 
& $\begin{array}{c}\text { Steady-state Moment } \\
(\mathbf{N m m})\end{array}$ & $\begin{array}{c}\text { Peak Moment } \\
(\mathbf{N m m})\end{array}$ & $\begin{array}{c}\text { Peak Angle } \\
(\mathbf{d e g})\end{array}$ \\
\hline Experimental & 100 & 2150 & 8.8 \\
Simulation & 18 & 279 & 9.1 \\
\hline
\end{tabular}

Table 3. Comparison of simulated and experimental moment-angle responses for single-matrix boom

Overall, it was found that this model of the boom is stable and allows for fast simulation times. However, it over-predicts the fold radius by $32 \%$. It is possible to extract a moment-angle curve that is qualitatively accurate but cannot reproduce the high peak moment value observed experimentally. These inaccuracies can stem from two sources. First, the *Tie constraints limits the shear transmission between the two tapesprings at larger fold angles which. Second, shell elements model the mid-plane of the composite and the *Tie constraint is hence applied at the mid-plane of the shell. In the prototype however, the mid-planes are not constrained to be coincident and the two tape-springs are separated by a shell thickness when fully folded. 


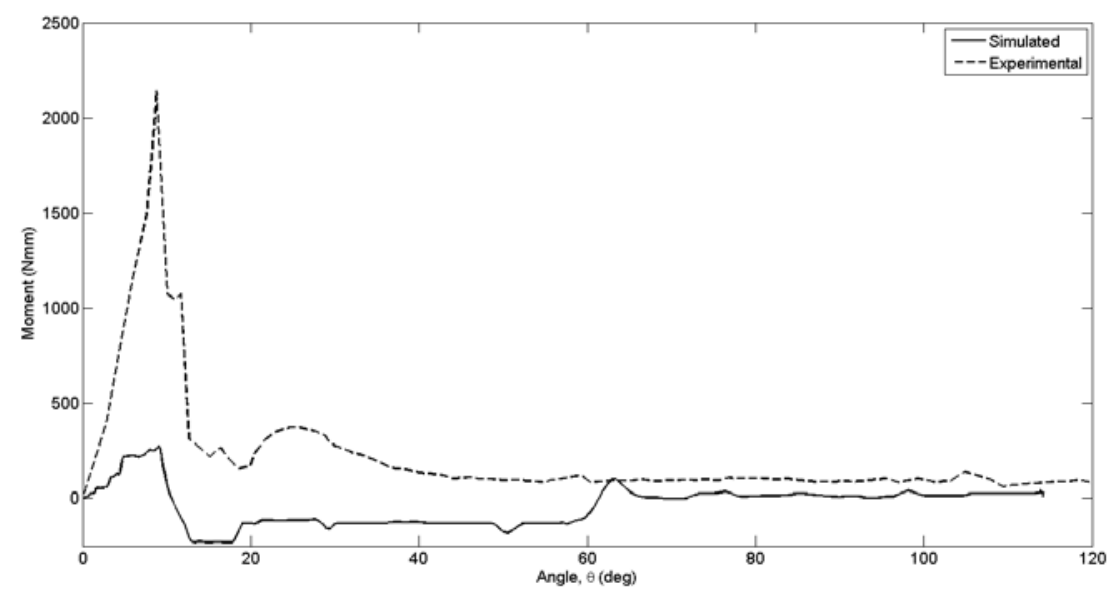

Figure 17. Simulated moment-angle curve

\section{Membrane Hinge Model}

An alternative model was set up by joining the two tape-springs by two rows of M3D3 membrane elements which have no bending stiffness. Each row is $0.1 \mathrm{~mm}$ in width, half the thickness of the shell. This allows the mid-planes of the two shells to be separated by a single shell thickness, as in the experiments. The in-plane shear in the connection is controlled through the shear compliance of the M3D3 elements.

The small width of the membrane elements results in an extremely fine mesh not only along the hinge but for the whole boom in order to maintain a maximum element aspect ratio of 3. S4 shell elements were used in order to reduce the total number of elements and speed up simulation. The total number of elements in this model was approximately, 73,600, 13 times that of the previous model. The small mesh size results in extremely long simulation times and more importantly causes stability issues as the step size required for stability is directly proportional to the minimum element length (see Equation 4).

The viscous damping was tuned in these simulations in a manner identical to the ${ }^{*}$ Tie model. The optimal damping value was chosen to be $c_{v}=2.0 \times 10^{-2} \mathrm{~kg} \cdot \mathrm{mm}^{-1} \cdot \mathrm{s}^{-1}$ for the folding step. The variation in damping values between the two models highlights once again the importance of carefully selecting the $c_{v}$ parameter.

This model results in a fold radius of $10.6 \mathrm{~mm}$, see Figure 18, only a $1.9 \%$ difference from the experimentally obtained value. This demonstrates the importance of the shell separation to the folded shape of the boom. However, deployment simulations still resulted in the fold not latching during deployment and stability issues of the model prevented further investigation of the deployment parameters.

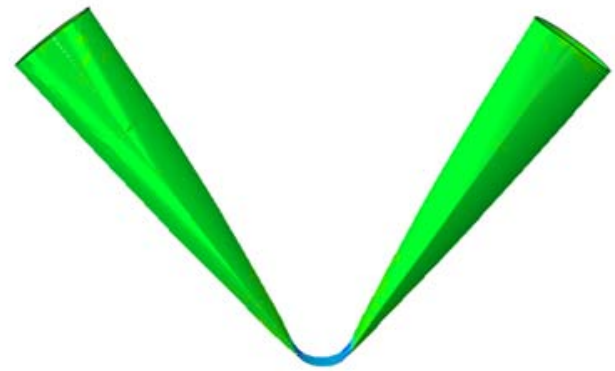

Figure 18. Folded shape obtained using model with M3D3 hinge elements. 


\section{Dual-Matrix Booms}

Following the simulation technique described in Section V.A, a finite element model of the dual-matrix composite boom was created. The silicone hinge was modeled using $8 \mathrm{~mm}$ wide regions of $\mathrm{S} 4$ shell elements. The model consisted of 10,577 nodes and 10,536 shell elements with a minimum element length of around $0.75 \mathrm{~mm}$.

In the case of dual-matrix composite booms, applying a local surface pressure load for pinching lead to a kink in the boom instead of the smooth fold region seen experimentally. Hence the boom was pinched with contact forces exerted by two rigid cylinders. The boom was then folded by smoothly applying a $90^{\circ}$ rotation at the dummy node $\mathrm{C}$ over $1.5 \mathrm{~s}$. The contact between the rigid cylinders and the boom was removed after folding to obtain the freestanding fully folded configuration by using the Contact Exclusions parameter. Figure 19 shows snapshots of different stages of folding.

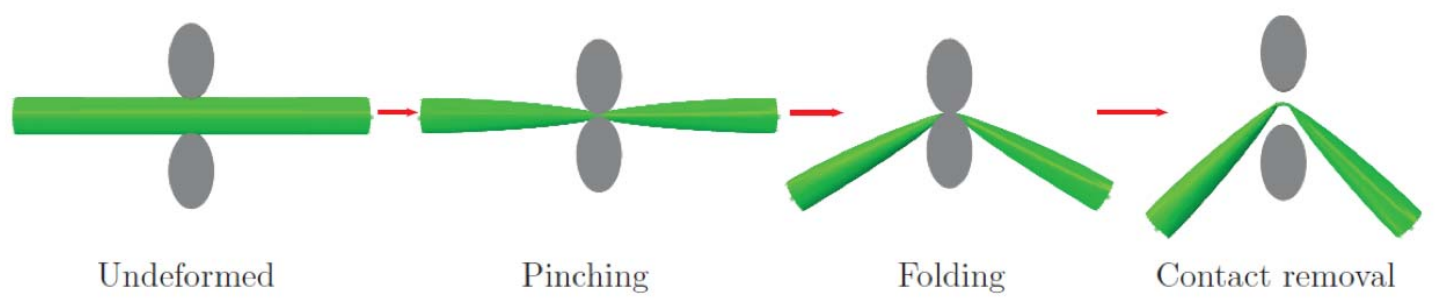

Figure 19. Folding sequence for dual-matrix composite boom.

Figure 20 shows two snapshots just before and soon after removing the contact between the boom and the rigid cylinders. Note that the boom forms a kink as soon as the rigid cylinders are removed. Refinement of the mesh did not show any significant effect on this behaviour.

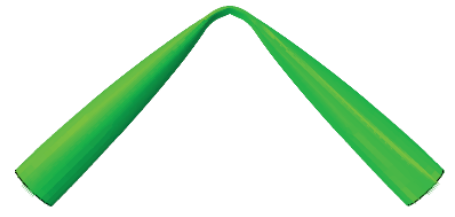

(a) before contact removal

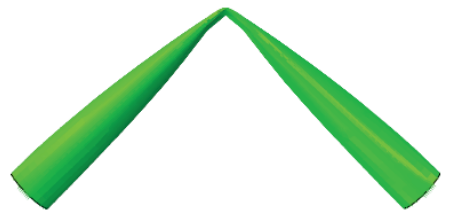

(b) after contact removal

Figure 20. Comparison of deformed shape before and after contact removal

Careful examination of the deformed shape of the boom cross-section, Figure 21, shows that the silicone hinge is reluctant to flatten and pushes back the tape-springs and hence forces the boom to form a kink at the centre.

To investigate the kink formation, the sensitivity to transverse shear stiffness was examined by varying three transverse shear coefficients for the AQ/silicone composite defined through the ${ }^{*}$ Transverse Shear parameter with the *Shell General Section keyword. Table 4 shows the parameters used in the sensitivity study. Note that model 1 is consistent with the default transverse shear coefficients used by the solver. The coefficients were reduced by an order of magnitude at a time and the folded shape was observed. Figure 22 shows the corresponding fully folded configurations obtained through these simulations. Model 4 showed a similar folded configuration to the experimental observations and therefore it was selected for further investigation of deployment behavior. Figure 23 shows the variation of the deformed cross-section at different location of the boom when it is folded to $90^{\circ}$. Note that boom cross-section is almost flat even $5 \mathrm{~mm}$ away from the center whereas the cross-section of previous model (see Figure 21) was not flat even $1 \mathrm{~mm}$ away from the center.

Model 4 resulted in a fold radius of $9.8 \mathrm{~mm}$, only a $2.0 \%$ difference from experiments. Figure 24 shows the simulated moment-rotation response observed. Though the curve follows a qualitatively similar pattern to the experimentally observed data, the predictions are significantly lower than the measured values (see Table 5). However, a good correlation was observed between simulation and experiments when it comes to deformed configuration, Figure 25. 


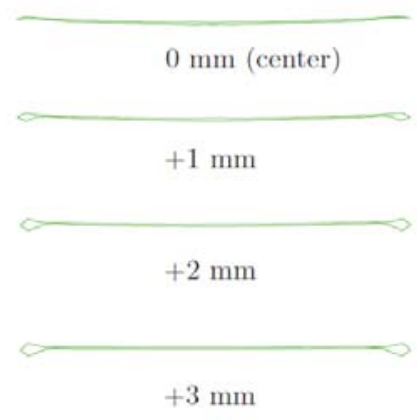

before contact removal

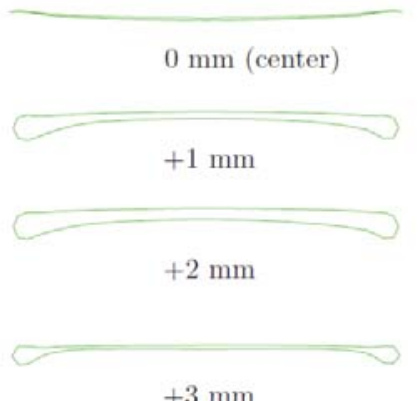

after contact removal

Figure 21. Comparison of deformed boom cross-sections before and after removing contact.

\begin{tabular}{ccccc} 
Model No. & $\mathbf{1}$ & $\mathbf{2}$ & $\mathbf{3}$ & $\mathbf{4}$ \\
\hline $\mathrm{K}_{11}$ & 918 & 91.8 & 9.18 & 0.918 \\
$\mathrm{~K}_{22}$ & 918 & 91.8 & 9.18 & 0.918 \\
$\mathrm{~K}_{12}$ & 0 & 0 & 0 & 0 \\
\hline
\end{tabular}

Table 4. Transverse shear coefficients for hinge region (AQ/silicone composite)

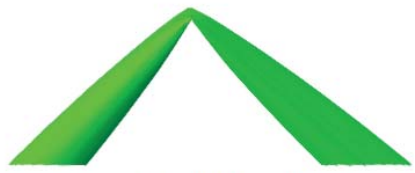

Model No. 1

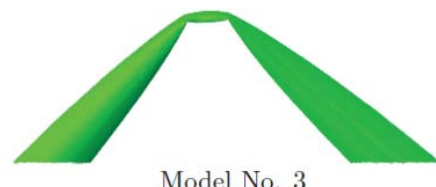

Model No. 3

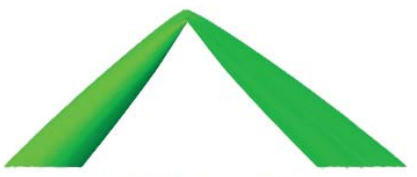

Model No. 2

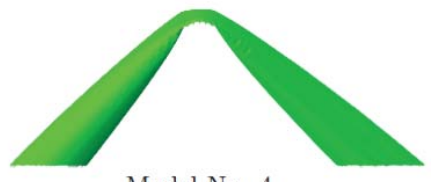

Model No. 4

Figure 22. Comparison of fully folded configurations with varying transverse shear coefficients given in Table 4

\begin{tabular}{rccc} 
& $\begin{array}{c}\text { Steady-state Moment } \\
(\mathbf{N m m})\end{array}$ & $\begin{array}{c}\text { Peak Moment } \\
(\mathbf{N m m})\end{array}$ & $\begin{array}{c}\text { Peak Angle } \\
(\mathbf{d e g})\end{array}$ \\
\hline Experimental & 247 & 2850 & 6.0 \\
Simulation & 19 & 304 & 4.7 \\
\hline
\end{tabular}

Table 5. Comparison of moment-angle relations for tape-spring and dual-matrix booms 


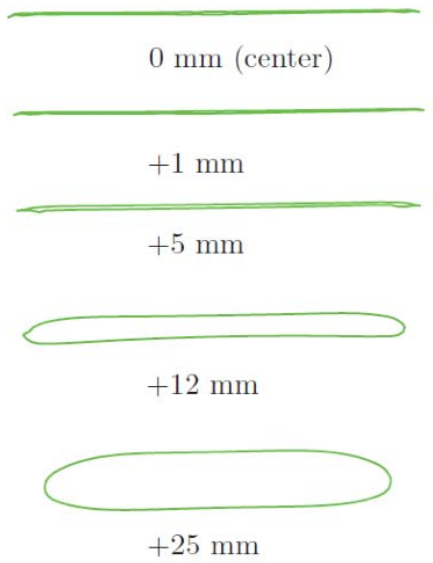

Figure 23. Variation of deformed boom cross-sections for Model 4

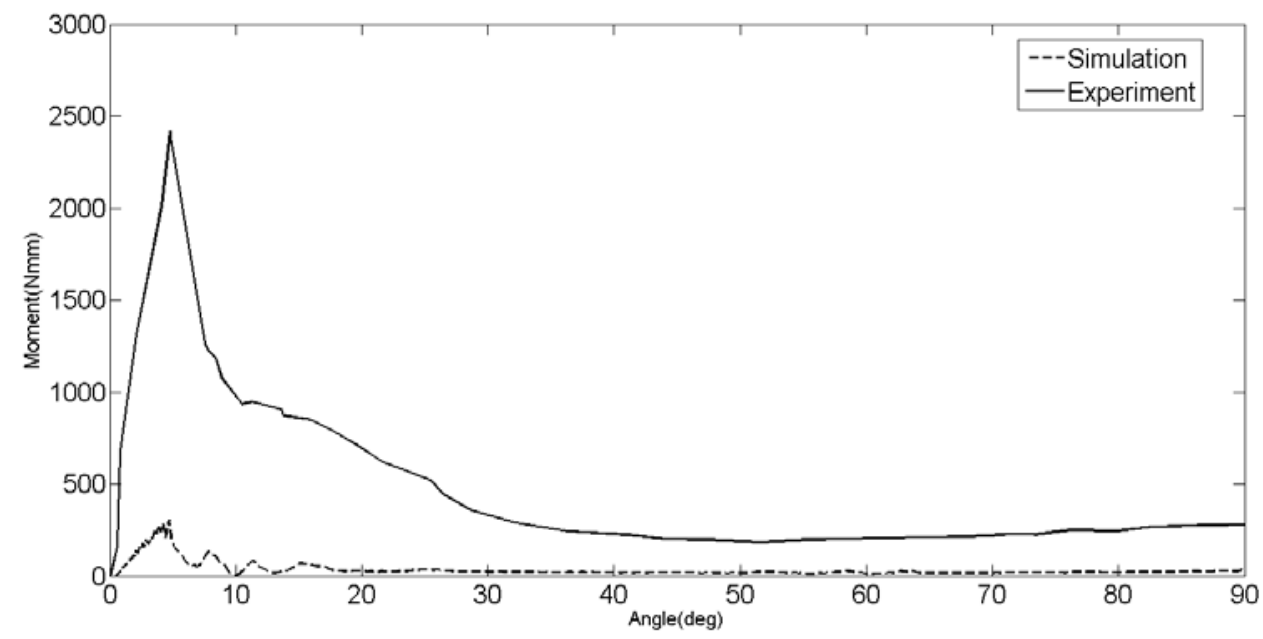

Figure 24. Simulated moment-angle curve for dual-matrix composite boom 


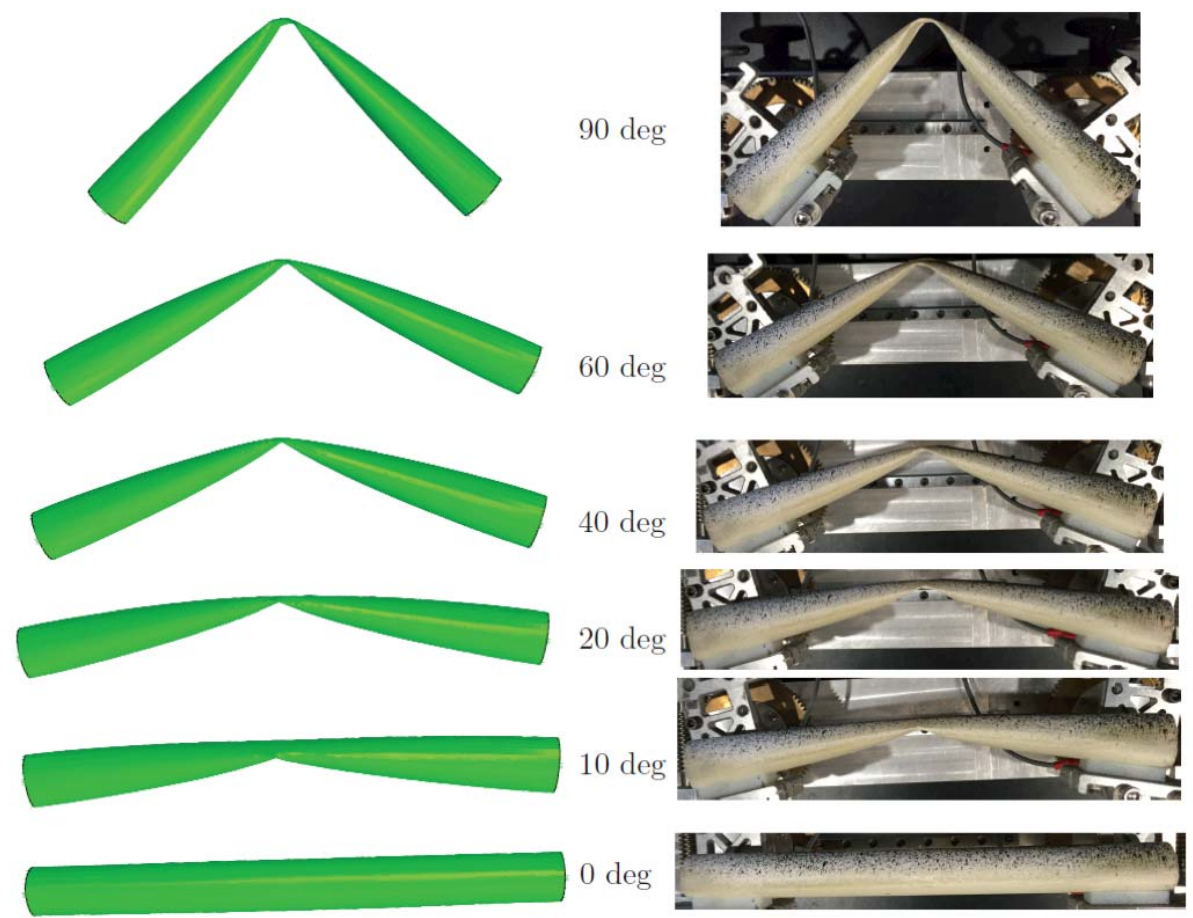

Figure 25. Comparison of dual-matrix boom configuration during deployment

As with the model for the single-matrix boom, the simulated steady-state value is close to that predicted theoretically. In fact, the whole simulated moment-rotation response is similar to that simulated for the single-matrix boom. Even though the dual-matrix boom is modeled as a single part, ABAQUS still does not capture the effects of the closed cross-section.

\section{Conclusion}

This work proposes dual-matrix composites as a way of realizing deployable closed cross-section booms that are stiff, lightweight, and can be packaged in small volumes. Little work exists on the folding and deployment of closed cross-section booms so this paper focused on developing experimental and simulation techniques for addressing this gap. The quasi-static behavior of a single fold at the center of a dual-matrix boom was investigated and compared to a single-matrix boom composed of two AQ/epoxy tape-springs. Both types of booms showed a moment-rotation response typical of a pair of tape-springs. It was demonstrated that the silicone hinges in the dual-matrix boom significantly contribute to the deployment moment of these structures. Furthermore, shape measurements of the fold regions using digital image correlation revealed a localized fold region of approximately a $10 \mathrm{~mm}$ radius for both booms. Experiments show that dualmatrix booms are stiff structures with a high peak deployment moment that exhibit reliable latching during deployment in samples with no fabrication defects.

Finite element models of both booms have been developed and identified viscous damping as a critical parameter in the quasi-static modeling of shell structures undergoing large deformations. The simulations captured the $10 \mathrm{~mm}$ fold radius of both booms with an error of $2 \%$ or less. However, the simulated momentrotation response, while qualitatively accurate, consistently under-predicted the peak moment and steadystate values measured experimentally. It was demonstrated that the simulated moment-rotation response for the two booms is similar to theoretical predictions for a pair of unattached tape-springs, implying that commercially available finite element software cannot accurately capture the significant contribution of the closed cross-section. This may be a result of the limited number of shell element types available in the software. 
Future work includes repeating simulations using a different finite element software with a larger selection of elements to develop techniques to successfully predict the deployment moment of closed cross-section booms. Furthermore, this work will be extended to an investigation of the dynamic deployment behavior of dual-matrix booms to evaluate the capability of these booms to deploy payloads on orbit.

\section{Acknowledgments}

This research was supported by the AFOSR (award no. FA9550-13-1-0061, program manager Dr David Stargel).

\section{References}

${ }^{1}$ M.W. Thomson. The Astromesh Deployable Reflector. Antennas and Propagation Society International Symposium, July 1999 (3):1516-1519, IEEE.

${ }^{2}$ Northrop Grumman. STEM Products and Programs. http://www.northropgrumman.com/BusinessVentures/ AstroAerospace/Products/Documents/pageDocs/STEM_Hardware_Programs.pdf, 2015.

${ }^{3}$ C. Sickinger, L. Herbeck, T. Strohlein, and J. Torrez-Torres. Lightweight Deployable Booms: Design, Manufacture, Verification, and Smart Materials Application. 55th International Astronautical Congress, IAF/IAA/IISL, Oct 04-08, 2004, Vancouver, Canada.

${ }^{4}$ F. Lopez Jimenez, and S. Pellegrino. Folding of fiber composites with a hyperelastic matrix. International Journal of Solids and Structures, Vol. 49, February 2012, pp. 395-407.

${ }^{5}$ T. W. Murphey, T. Meink, and. M. M. Mikulas. Some micromechanics considerations of the folding of rigidizable composite materials. 42nd AIAA/ASME/ASCE/AHS/ASC Structures, Structural Dynamics, and Materials Conference, AIAA $2001-1418$.

${ }^{6}$ I. Maqueda, S. Pellegrino, and. J. M. Majia-Ariza. Characterization of a high strain composite material. 53rd AIAA/ASME/ASCE/AHS/ASC Structures, Structural Dynamics, and Materials Conference, 23-26 April 2012 Honolulu, Hawaii.

${ }^{7}$ K. Saito, S. Pellegrino, and. T. Nojima. Manufacture of arbitrary cross-section composte honeycomb cored based on origami techniques. Proceedings of the ASME 2013 IDETC/CIE, Portland, OR, August 2013, American Society of Mechanical Engineering.

${ }^{8}$ M. Sakovsky, I. Maqueda, C. Karl, S. Pellegrino, and. J. Costantine. Dual-Matrix Composite Wideband Antenna Structures for CubeSats. AIAA Spacecraft Structures Conference, 5-9 January 2015, Kissimmee, FL, AIAA 2015-0944.

${ }^{9}$ I. Maqueda. High-Strain Composites and Dual-Matrix Composite Structures. PhD Thesis, California Institute of Technology, 2014.

${ }^{10}$ P. Reu. The Art and Application of DIC. Stereo-rig Design: Stereo-Angle Selection. Experimental Techniques, Vol. 37, 2013, pp. 1-2.

${ }^{11}$ H.M.Y.C. Mallikarachchi, and S. Pellegrino. Design of ultrathin composite self-deployable booms, Journal of Spacecraft and Rockets, Vol. 51(6), 2014, pp. 1811-1821.

${ }^{12}$ O. Soykasap., Micromechanical Models for Bending Behavior of Woven Composites, Journal of Spacecraft and Rockets, Vol. 43, No. 5, pp. 1093-1100, 2006.

${ }^{13}$ S. Pellegrino. Folding and Deployment of Thin Shell Structures, (In: Extremely Deformable Structures) (edited by D. Bigoni). Springer: pp. 1-89, 2015. 